\title{
Design of Reverse Osmosis Seawater Desalination Control System Based on LADRC
}

\author{
Hongli Liu a, Shijia Liu ${ }^{b}$, Lei Shao ${ }^{c}$, Ji Li c , Xiaoqi Chen ${ }^{c}$ \\ School of Electrical and Electronic Engineering, Tianjin University of Technology, Tianjin 300384, \\ China. \\ a984875777@qq.com, b971439166@qq.com, c1302023387@qq.com
}

Keywords: Ro, LADRC control, LESO, Energy consumption problem, Liapunov's stability.

\begin{abstract}
According to the complexity of parameters in reverse osmosis desalination system, coupling, nonlinear, time-varying, hysteresis and other characteristics, this paper uses linear active disturbance rejection control (LADRC) to control the temperature and pressure, (because temperature and pressure make a big difference on the equipment and water quality), it aims to reduce the energy consumption of the system. In this paper, the constant temperature and pressure water supply system based on LADRC is established respectively for temperature and pressure. Compared with traditional PID control, the simulation results show that LADRC control is faster than the PID control response, with steady-state precision, better anti-interference ability. At the same time, the stability of second order linear expansion state observer (LESO) in the first order LADRC is proved by the Lyapunov stability method. The application of LADRC control in reverse osmosis control system can realize the real-time tracking of input to output, and make the system in steady state control better, and when the system is in steady state, the consumption of equipment is lowest, thus reducing system energy consumption, which has important practical value and research significance.
\end{abstract}

\section{Introduction}

Reverse osmosis technology is a pressure-driven membrane separation process ${ }^{[1]}$. Although this process of energy consumption has a great advantage over other desalination technology, its energy consumption costs still account for $70 \%$ of the total cost of the system. Cost and investment costs are too high, which has been regarded as the main problem the desalination faces, and energy consumption is the key to determine its cost. To reduce energy consumption, save energy, reduce the cost of water has become the consensus of industry field ${ }^{[2]}$.

In the reverse osmosis desalination system, the power consumption of high-pressure pumps accounts for about $35 \%$ of the operating costs of the system, which is one of the main factors affecting the cost of water ${ }^{[3]}$. And the pressure is the key variable to control the start and stop of high pressure pump, the filtering process of the filter at all levels during the pre-processing, the switch of the valve and so on will cause the pressure change in the system pipeline. The pressure change makes the high pressure pump start and stop frequently, which cause a large amount of energy consumption, and affect the life and quality of the high-pressure pump, and cause great damage to the reverse osmosis membrane, so we need to design a constant pressure control system. The temperature affects the osmotic pressure, thus affecting the pressure difference, so it is necessary to find a suitable temperature, which makes the high-pressure pump and heating system have the lowest energy consumption, so it is necessary to design a constant temperature control system ${ }^{[4]}$.

The conventional control algorithm has the traditional PID control, the control method is simple, but it can not achieve a good control effect for the large time-delay system such as temperature. And the combination of PID and intelligent algorithms, such as fuzzy control algorithm ${ }^{[5]}$, genetic algorithm $(\mathrm{GA})^{[6]}$, BP neural network algorithm ${ }^{[7]}$, and so on, the steady state accuracy of these control algorithms and the disturbance control effect of disturbance are not good. In this paper, LADRC control algorithm is used to achieve good steady-state control of temperature and pressure ${ }^{[8]}$, and the 
effect of anti-interference is strong, so as to provide a guarantee for stable and efficient operation of the system $^{[9]}$.

\section{Design and simulation based on LADRC controller}

\subsection{LADRC basic control structure and principle.}

LADRC inherits the idea that ADRC uses error feedback to control ${ }^{[10]}$.Compared with ADRC, LADRC eliminates the tracking differentiator, and the state error feedback adopts the linear combination form, and the linear extended state observer (LADRC) is used to estimate the total disturbance of the system.

Figure 1 is a closed loop control system consisting of first-order LADRCcontroller. The controlled object is a single input single output structure, $k_{p} 、 b_{0}$ and $\omega_{0}$ in the LESO are three parameters that need to be set. LESO is in the form of state space equations defined as follows:

$$
\left\{\begin{array}{l}
A=\left[\begin{array}{ll}
-2 \omega_{0} & 1 \\
-\omega_{0}{ }^{2} & 0
\end{array}\right] \\
B_{s}=\left[\begin{array}{cc}
b_{0} & 2 \omega_{0} \\
0 & \omega_{0}^{2}
\end{array}\right] \\
C_{s}=I_{2 \times 2} \\
E=0_{2 \times 2}
\end{array}\right.
$$

By adjusting $\mathrm{k}_{\mathrm{p}}, \omega_{0}$ and $\mathrm{b}_{0}$, the BIBO stability of the whole system can be guaranteed for the system with the first order of the controlled object.

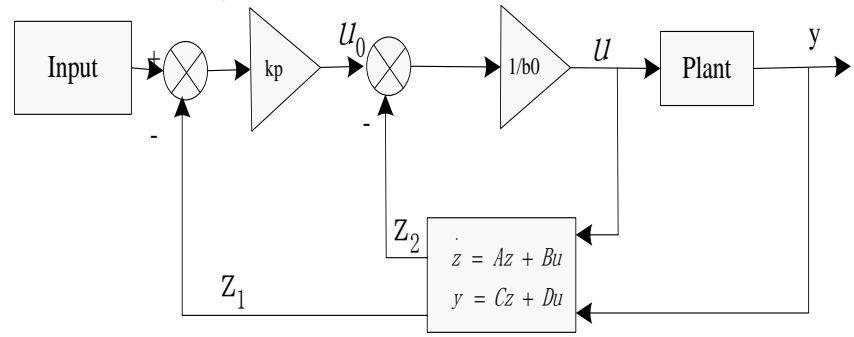

Fig1: First order LADRC controller

\subsection{Design of reverse osmosis constant pressure water supply system based on LADRC.}

The reverse osmosis system in water pump with constant pressure water supply scheme can reduce the raw water pump and high pressure pump frequent start and stop control, reduce power consumption, reduce the risk of accidents, prolong the service life of the high-pressure pump, thereby reducing the energy consumption of the system. Constant pressure water supply system is to supply raw water constant pressure to pretreatment device, thus providing stable water source for high pressure pump. The control loop of the whole system is shown in Fig2.

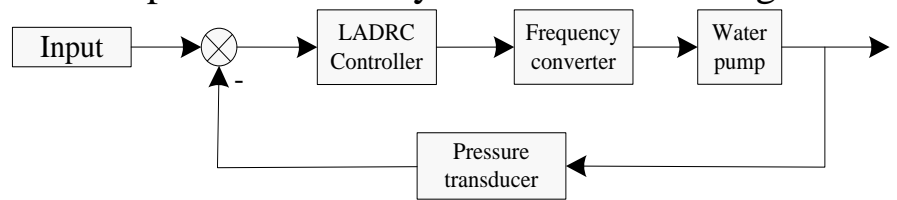

Fig2: Constant voltage control loop

In the constant pressure water supply process, when the system is started, the pressure is close to zero, which is a pure delay process. As the pressure continues to rise, the water gradually fills the whole pipe and tank, and the pressure increases gradually until it reaches a steady level, which can be considered as a first order inertial link. Therefore, the mathematical model of the water pump pipe system can be equivalent to a first-order inertial link with pure hysteresis. That is:

$$
G(s)=\frac{K_{w}}{T_{w} s+1} e^{-\tau s}
$$


where: $\mathrm{K}_{\mathrm{w}}$ represent gain, $\mathrm{T}_{\mathrm{w}}$ represent inertia time constant, $\tau$ represent pure delay time.

\subsection{Design of reverse osmosis constant temperature water supply system based on LADRC.}

It is known by osmotic pressure $\Pi=R T \sum c_{i}$, the temperature effects of osmotic pressure, thus affecting the pressure difference, if the water temperature of reverse osmosis is too low or too high will make the membrane system pressure increases, on the premise of ensuring stable and efficient production of water, it is necessary to find the best running water temperature to minimize the total consumption of the high-pressure pump and heating system. In order to meet this goal, at its outlet temperature with heat exchanger, using high temperature steam heating treatment of raw water, in order to better control the heating temperature, the steam outlet is provided with steam control valve to control the size of steam.

If the traditional PID controller is used to control the controlled quantity, the control system can not meet the control requirement because the temperature dalay time is long. For such a system with large time delay, the LADRC controller can effectively reduce the influence of time delay on the system, and no precise mathematical model is needed. Therefore, the LADRC controller is more applicable to such industrial control systems that are complex, what's more, the exact and precise process model of the system cannot be determined.

The system control loop is as follows:

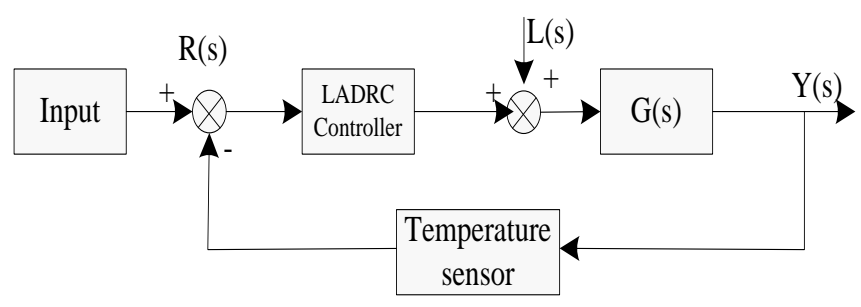

Fig3:Constant temperature control circuit

The steam temperature heat transfer process is abstracted into a first-order inertia link,

$G_{(s)}=\frac{K_{w}}{T_{w} s+1} e^{-\tau s}$

\subsection{Stability analysis.}

From Fig1, it can be seen that the linear ADRC is a closed loop and has stability problem. For the first order linear ADRC model, the stability is proved in the case of disturbance is given.

The linear extended state observer of the system is

$\left\{\begin{array}{l}\dot{z_{1}}=z_{2}+L_{1}\left(y_{1}-\hat{y}_{1}\right)+b_{0} u \\ z_{2}=L_{2}\left(y_{1}-\hat{y}_{1}\right)+h(\hat{y}, w) \\ \hat{y}=z_{1}\end{array}\right.$

According to the above, the gain is selected as $L=\left[\begin{array}{c}\beta_{1} \\ \beta_{2}\end{array}\right]=\left[\begin{array}{c}a_{1} \omega_{0} \\ a_{2} \omega_{0}{ }^{2}\end{array}\right]=\left[\begin{array}{c}2 \omega_{0} \\ \omega_{0}{ }^{2}\end{array}\right]$,

Let $\tilde{y}_{i}=y_{i}-\hat{y}_{i}, \mathrm{i}=1,2$, the estimation error of the extended state observer is derived from the formula (1)

$\left\{\begin{array}{l}\dot{\tilde{y}}_{1}=\tilde{y}_{2}-2 \omega_{0} \tilde{y}_{1} \\ \dot{\tilde{y}}_{2}=h(y, w)-h(\hat{y}, w)-\omega_{0}^{2} \tilde{y}_{1}\end{array}\right.$

Let $\varepsilon_{j}=\frac{\tilde{y}_{j}}{\omega_{0}{ }^{j-1}}, \mathrm{j}=1,2$, the formula (2) can be changed to 


$$
\left\{\begin{array}{l}
\dot{\varepsilon}_{1}=\omega_{0} \varepsilon_{2}-2 \omega_{0} \varepsilon_{1} \\
\dot{\varepsilon}_{2}=-\omega_{0} \varepsilon_{1}+\frac{h(y, w)-h(\hat{y}, w)}{\omega_{0}}
\end{array}\right.
$$

that is $\dot{\varepsilon}_{j}=\omega_{0}\left[\begin{array}{ll}-2 & 1 \\ -1 & 0\end{array}\right]\left[\begin{array}{l}\varepsilon_{1} \\ \varepsilon_{2}\end{array}\right]+\left[\begin{array}{l}0 \\ 1\end{array}\right] \frac{h(y, w)-h(\hat{y}, w)}{\omega_{0}}$. Let $A_{0}=\left[\begin{array}{ll}-2 & 1 \\ -1 & 0\end{array}\right], B_{0}=\left[\begin{array}{l}0 \\ 1\end{array}\right]$, as we know above, LESO's double pole assignment is in $-\omega_{0}$, thus $\left[\begin{array}{ll}-a_{1} & 1 \\ -a_{2} & 0\end{array}\right]$ is Hurwitz stable. Then there is a positive definite Hemet matrix, make $A_{0}$ satisfy $A_{0}^{T} P+P A_{0}=-I$, and $P=\left[\begin{array}{cc}\frac{1}{2} & -\frac{1}{2} \\ -\frac{1}{2} & \frac{3}{2}\end{array}\right]$,

Defining Lyapunov function:

$$
\dot{V}(\varepsilon)=-\omega_{0}\left(\varepsilon_{1}^{2}+\varepsilon_{2}^{2}\right)+\frac{h(y, w)-h(\hat{y}, w)}{\omega_{0}}\left(-\varepsilon_{1}+3 \varepsilon_{2}\right)
$$

The $\mathrm{h}(\mathrm{y}, \mathrm{w})$ satisfies the Lipschitz continuity conditions in the domain, then there exists a constant $\mathrm{c}$ such that make $|h(y, w)-h(\hat{y}, w)| \leq c\|y-\hat{y}\|$, thus $\frac{|h(y, w)-h(\hat{y}, w)|}{\omega_{0}}$ can satisfty

$$
\frac{|h(y, w)-h(\hat{y}, w)|}{\omega_{0}}\left(-\varepsilon_{1}+3 \varepsilon_{2}\right) \leq c\left(-\varepsilon_{1}+3 \varepsilon_{2}\right) \frac{\|y-\hat{y}\|}{\omega_{0}}
$$

There is also $-\varepsilon_{1}+3 \varepsilon_{2}=2 \varepsilon^{T} P B_{0}$, so formula (5) can be changed to

$$
2 \varepsilon^{T} P B_{0} \frac{|h(y, w)-h(\hat{y}, w)|}{\omega_{0}} \leq 2 \varepsilon^{T} P B_{0} c \frac{\|y-\hat{y}\|}{\omega_{0}}
$$

When $\omega_{0} \geq 1$, there is $\frac{\|y-\hat{y}\|}{\omega_{0}}=\frac{\|\tilde{y}\|}{\omega_{0}} \leq\|\tilde{y}\|$, at the same time, because $\left\|\mathrm{PB}_{0} \mathrm{c}\right\|^{2}-2\left\|\mathrm{~PB}_{0} \mathrm{c}\right\|+1 \geq 0$, so

$$
2 \varepsilon^{T} P B_{0} \frac{|h(y, w)-h(\hat{y}, w)|}{\omega_{0}} \leq\left(\left\|P B_{0} c\right\|^{2}+1\right)\|\varepsilon\|_{1}^{2}
$$

It can be obtained from formula (6) (7)

$$
\dot{V}(\varepsilon) \leq-\omega_{0}\left(\varepsilon_{1}^{2}+\varepsilon_{2}^{2}\right)+\left(\left\|P B_{0}\right\|^{2}+1\right)\|\varepsilon\|_{1}^{2}
$$

When $\omega_{0} \geq\left\|\mathrm{PB}_{0} \mathrm{c}\right\|^{2}+1, \dot{V}(\varepsilon)<0$, according to the Lyapunov stability, there is $\lim _{t \rightarrow \infty} \tilde{y}_{i}(t)=0, \mathrm{i}=1,2$

The $\mathrm{P}$ control rate adopted by the LSEF, that is

$$
u_{0}=k_{p}\left(v-z_{1}\right)
$$

where: $\mathrm{k}_{\mathrm{p}}$ is proportionality constant; $\mathrm{v}$ is input value, Here, $\mathrm{u}_{0}$ is the error feedback control quantity, but has not added the compensation to the disturbance estimate. Considering the disturbance compensation, on the basis of the two order linear extended state observer designed above, the final control quantity can be taken as

$$
u=\frac{u_{0}-z_{2}}{b_{0}}
$$

from formula (10)(11),we can get

$$
\mathrm{u}=\frac{k_{p}\left(v-\hat{y}_{1}\right)-\hat{y}_{2}}{b_{0}}
$$

Let $\mathrm{e}=v-y_{1}$, we can get from formula 


$$
\begin{aligned}
& \mathrm{u}=\frac{k_{p}\left(\mathrm{e}+\hat{y}_{1}\right)-\left(y_{2}-\hat{y}_{2}\right)}{b_{0}} \\
& \dot{e}=\dot{v}-\dot{y}=\dot{v}-\left(y_{2}+b_{0} u\right)=-k_{p}\left(e+\tilde{y}_{1}\right)-\tilde{y}_{2}+\dot{v}
\end{aligned}
$$

In the whole LADRC, there is the TD (trackingdifferentiator), which is used to arrange the ideal transition process and give the differential signal of the transition process. But considering the delay of the object itself is slow, so consider removing the TD, hoping to start with a large error control signal, the object incentive, so that the output rushed out as soon as possible. Then the system becomes

$$
\dot{e}=-\mathrm{k}_{p}\left(e+\tilde{y}_{1}\right)-\tilde{y}_{2}
$$

Formula (15) is written in the form of state space:

$$
\dot{e}=\left[-\mathrm{k}_{\mathrm{p}}\right] \mathrm{e}(\mathrm{t})+\left[-\mathrm{k}_{\mathrm{p}}-1\right] \tilde{\mathrm{y}}(\mathrm{t})
$$

-kp makes the characteristic polynomial s-kp satisfy the Routh criterion, so [-kp] is Hurwitz stable, at the same time, the formula (9) shows $\lim _{t \rightarrow \infty}\left\|\left[-k_{p}-1\right] \tilde{y}(t)\right\|=0$, so $\lim _{t \rightarrow \infty}\|\mathrm{e}(\mathrm{t})\|=0$.

According to the Lyapunov asymptotic stability of the significance of the LADRC is asymptotically stable, but also on the stability of Engineering significance.For the stability analysis, the stability condition of LADRC is obtained, which provides a reliable theoretical basis for parameter tuning.

\subsection{Simulation results analysis}

\subsubsection{Constant pressure water supply system.}

According to the observation of the peak water consumption of the system, let $\mathrm{Kw}=1, \mathrm{Tw}=20, \tau=1$. Simulink is used to simulate the system, at the same time, compare with the traditional PID control. The simulation results are shown in Fig4.

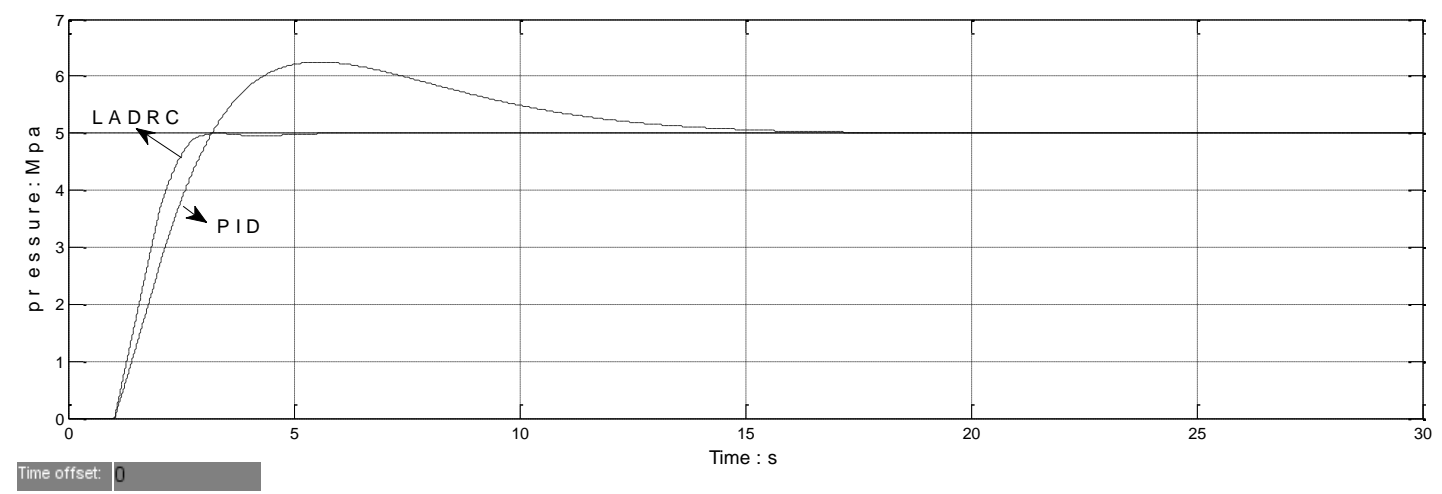

Fig4: Simulation curve of constant pressure water supply system

From the simulation results, it can be seen that the LADRC control is faster than the traditional PID control, without overshoot and no oscillation. In the actual operation of the system, the control scheme reduces the fluctuation of system pipe pressure, and meets the requirements of pressure control in high-pressure pump and other equipment in the system.

\subsubsection{Constant temperature water supply system.}

Set up the control system in SIMULINK, set $\mathrm{Kw}=1, \mathrm{Tw}=500$. The simulation results are shown in Fig5. 


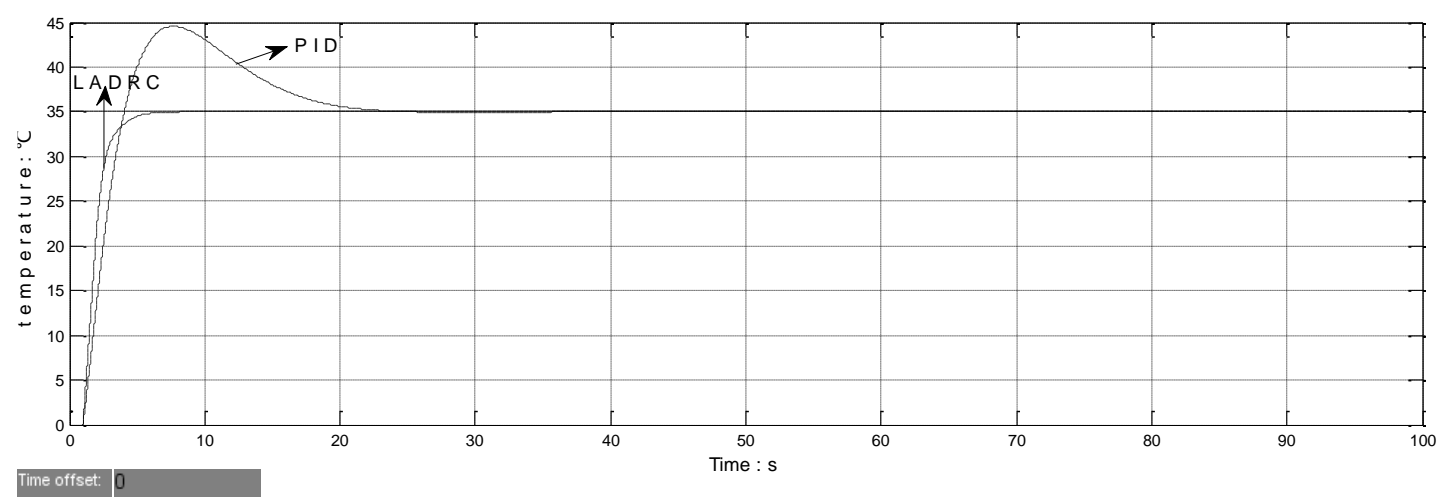

Fig5: Simulation curve of constant temperature system

Based on the simulation results, only the PID algorithm is used to control the temperature of the heat exchanger cannot achieve satisfactory control results, when the system adopts LADRC controller well overcome the influence of time delay on the system, the delay in the system is converted into time delay, it overcomes the drawbacks of the traditional PID control system which cannot control the large time delay system, and has achieved good results in the actual system operation.

\section{Summary}

For such a nonlinear, large delay, coupled complex industrial system as reverse osmosis desalination system, LADRC controller has better steady-state control of pressure and temperature, faster response and smaller steady-state error, which can make the system faster and more accurate to achieve steady-state, and avoid other energy consumption. And the LESO is used to estimate the disturbance in real time, and then it is used in feedback control, which will achieve rapid elimination of disturbance and it has strong anti-interference ability, thus can meet the reverse osmosis desalination of water temperature and pressure control requirements, which provides a guarantee not only for the stable operation of reverse osmosis seawater desalination system, but also for reducing the energy consumption of the system and it has a strong practical value.

\section{Acknowledgements}

This work was financially supported by Tianjin science and technology plan project (15ZXZNGX00140), Tianjin applied basic research project - Tianjin science and technology special commissioner project (16JCTPJC49400).

\section{References}

[1] Aipeng Jiang, Wen Cheng, Jian Wang, et al. Operation optimization of full flowsheet spiral-wound seawater reverse osmosis system. CIESC Journal, Vol. 65(2014) No. 4, p.1333-1343.

[2] A. Jiang, J. Wang, L.T. Biegler, et al. Operational cost optimization of a full-scale SWRO system under multi-parameter variable condi-tions, Desalination. Vol. 355 (2015), p. 124-140.

[3] Xiaoyan Ye, Guole $\mathrm{Xu}$, Jingning $\mathrm{Hu}$. The selection and optimization of high-pressure pump in reverse osmosis seawater desalination. Technology of Water Treatment, Vol. 34(2008) No. 9, p.79-81.

[4] C.P. Koutsou, A.J. Karabelas, M. Kostoglou, Membrane desalination under constant water recovery - the effect of module design parameters on system performance. Sep. Purif. Technol. Vol. 147 (2015), p. 90-113.

[5] Yang Zhao, Xinghua Qu, Rui Li, Self-adjusting control system of temperature based on fuzzy algorithm, Journal of Tianjin University, Vol. 44 (2011) No. 1, p.73-78. 
[6] Xiaoyun Fu, Xu Fang, Gang Yang, et al. Design and Simulation of GA-based PID controller. Journal of Huazhong University of Science and Technology (Natural Science Edition), Vol.40 (2012) No. 5, p.1-5.

[7] Yi Qu, Duo Ning, Zhanchi Lai, et cl. Neural networks based on PID control for greenhouse temperature. Transactions of the CSAE, Vol. 27(2011) No. 2, p. 307-311.

[8] Yi Sun, Zengjian Zheng, Jihong Dan, et cl. Parameters optimization of pressure and temperature based on the marine reverse osmosis desalination device. Journal of Zhejiang University of Technology. Vol. 44 (2016) No. 4, p. 359-363.

[9] Seung Joon Kim, Young Geun Lee, Sanghoun Oh, et cl. Energy saving methodology for the SWRO desalination process: control of operating temperature and pressure. Desalination. Vol. 247 (2009), p. 260-270.

[10] [10]. Gao Z Q. Active disturbance rejection control: A paradigm shift in feedback control system design[C]//Processdings of the American Control Conference. Piscataway, NJ:IEEE Press, Vol. 2006, p. 2399-2405. 